\title{
Observation of Tungsten Line Emissions in Wavelength Range of 10 - 500 Å in Large Helical Device*)
}

\author{
Yang LIU ${ }^{1)}$, Shigeru MORITA ${ }^{1,2)}$, Tetsutarou OISHI ${ }^{1,2)}$, Motoshi GOTO ${ }^{1,2)}$ and Xianli HUANG ${ }^{2)}$ \\ ${ }^{1)}$ Department of Fusion Science, Graduate University for Advanced Studies, Toki, Gifu 509-5292, Japan \\ 2) National Institute for Fusion Science, Toki, Gifu 509-5292, Japan
}

(Received 28 December 2017 / Accepted 19 February 2018)

\begin{abstract}
Tungsten spectra have been observed in Large Helical Device (LHD) by injecting a coaxial tungsten pellet

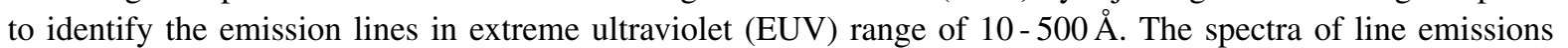
from tungsten ions have been measured in neutral-beam-heated discharges using two EUV spectrometers of EUV_Short and EUV_Long working in wavelength ranges of $10-130 \AA$ and $50-500 \AA$, respectively. As a result, a lot of tungsten lines from low-ionized ions of $\mathrm{W}^{4+}, \mathrm{W}^{6+}$ and $\mathrm{W}^{7+}$ are observed for the first time in the toroidal device in addition to tungsten lines from highly ionized ions of $\mathrm{W}^{41+}-\mathrm{W}^{45+}$. Measured line emissions are carefully identified based on the NIST database and the determined wavelengths show a good agreement with the NIST database. The result is summarized in table with information on blended lines obtained from the radial profile measurement.
\end{abstract}

(c) 2018 The Japan Society of Plasma Science and Nuclear Fusion Research

Keywords: tungsten spectra, extreme ultraviolet, spectrometer, pellet injection, Large Helical Device

DOI: $10.1585 /$ pfr. 13.3402020

\section{Introduction}

In ITER (International Thermonuclear Experimental Reactor), which is a next-generation device for fusion research carrying out D-T burning plasma experiments, the tungsten material is used for plasma facing components (PFCs) in divertor region, since several positive characteristics are confirmed in the tungsten, i.e. high melting point, low sputtering yield and low tritium retention $[1,2]$. A spectroscopic study of tungsten becomes then important for understanding of the tungsten transport in core and edge plasmas and accurate determination of the radiative cooling rate.

A neutral tungsten emission, $\mathrm{W}_{\mathrm{I}}$, observed in visible range at $4009 \AA[3,4]$ has been now widely used for the plasma wall interaction study $[5,6]$. Until now, any other lines from low ionized tungsten ions for the study of tungsten behavior in plasma edge have not been observed in the visible range above $4000 \AA$ due to a lack of sufficient intensity. Line emissions from weakly ionized tungsten ions, $\mathrm{W}_{\text {IV-VII }}$, have been observed in vacuum ultraviolet (VUV) range of $500-1500 \AA$ using a $3 \mathrm{~m}$ space-resolved VUV spectrometer with good spectral resolution [7]. The $\mathrm{W}_{\mathrm{XLV}}$ $\left(\mathrm{W}^{44+}\right)$ and $\mathrm{W}_{\mathrm{XLVI}}\left(\mathrm{W}^{45+}\right)$ emissions have been already observed from many devices in extreme ultraviolet (EUV) range in the vicinity of $60 \AA$ and $130 \AA[8,9]$. However, the spectral investigation of tungsten line emissions over the whole EUV wavelength range is still insufficient. In particular, tungsten spectral lines emitted in longer wavelength

author'se-mail: liu.yang@nifs.ac.jp

*) This article is based on the presentation at the 26th International Toki Conference (ITC26). range, e.g. 200 - $500 \AA$, are not well examined because it is believed that tungsten lines probably do not exist in such a long wavelength range, while a soft $\mathrm{x}$-ray spectrometer observing the wavelength range of $150-400 \AA$ is planned to install on ITER for divertor diagnostics [10]. In order to accurately identify the tungsten spectral line over the entire EUV wavelength range, observations have been made not only for wavelength spectra but also for radial profiles in Large Helical Device (LHD) by injecting a coaxial graphite pellet with a small amount of tungsten [11]. Since LHD does not need any plasma current for plasma confinement, discharges are entirely tolerant for the impurity buildup. Therefore, the pellet injection with a variety of tungsten concentrations is possible in LHD. As a result, it is possible to generate a bright tungsten light source based on the LHD discharge with neutral beam injection (NBI). As the electron temperature of neutral-beam heated plasmas in LHD typically ranges in $1 \mathrm{keV} \leq T_{\mathrm{e}} \leq 4 \mathrm{keV}$, most of line spectra from tungsten ions are emitted in the EUV range of $10-500 \AA$. The tungsten spectra in $10-500 \AA$ are carefully identified and the result is summarized in a table. Several line emissions from low-ionized tungsten ions are newly found in longer wavelength range of $200-500 \AA$.

\section{Experimental Setup}

Two grazing-incidence EUV spectrometers called EUV_Short [12] and EUV_Long [13] are used for the present study to observe tungsten line emissions in the wavelength range of $10-130 \AA$ and $50-500 \AA$, respectively. Both spectrometers have identical mechanical structures except for the grating and angle of incidence, 
i.e. a fixed entrance slit width of $30 \mu \mathrm{m}$, a gold-coated concave varied-lines-spacing (VLS) laminar-type holographic grating and a back-illuminated charge-coupled device (CCD: $1024 \times 255$ pixels, $26 \times 26 \mu \mathrm{m}^{2} /$ pixel). The VLS grating with 2400 grooves/mm (1200 grooves $/ \mathrm{mm}$ ) for EUV_Short (EUV_Long) requires the angle of incidence of $88.6^{\circ}\left(87.0^{\circ}\right)$. The wavelength spectrum is sequentially obtained at each $5 \mathrm{~ms}$ interval with full-binning mode in CCD. Both spectrometers are installed on \#10-O port at a distance of $\sim 10 \mathrm{~m}$ away from the plasma center and the tungsten pellet is also injected at the same port.

A space-resolved EUV spectrometer called EUV Long2 [14] is installed for impurity profile measurement at wavelength range of 50-500 $\AA$. The long axis of CCD is set perpendicular to the wavelength dispersion direction for the vertical profile measurement. The CCD is routinely operated in sub-image mode with five-pixel binning in the long axis and two-pixel binning in the short axis. The resultant CCD output signal shows an image of vertical profile versus wavelength with size of $204 \times 127$ channels. The temporal resolution of EUV_Long2 is generally $100 \mathrm{~ms}$. The total vertical observation range in the profile measurement is nearly $0.5 \mathrm{~m}$ of which the range corresponds to half the vertical plasma diameter at the horizontally elongated plasma cross section.

\section{Experimental Results}

A series of experiments on the tungsten spectroscopic study have been done in LHD with tungsten pellet injection. A coaxial-structured tungsten pellet with a thin tungsten wire inserted into a polyethylene or graphite cylindrical tube is injected to NBI hydrogen discharges with speed of $\sim 200 \mathrm{~m} / \mathrm{s}$. The tungsten spectra in EUV range are measured in hydrogen discharges at magnetic axis position of $R_{\mathrm{ax}}=3.6 \mathrm{~m}$ and toroidal magnetic field of $B_{\mathrm{T}}=-2.75 \mathrm{~T}$. The minus sign of $B_{\mathrm{T}}$ means magnetic field in counterclockwise direction.

A typical discharge with tungsten pellet injection is shown in Fig. 1. The pellet is injected at $t=4.33 \mathrm{~s}$. As the tungsten wire with $0.05 \mathrm{~mm}$ diameter and $0.7 \mathrm{~mm}$ length is used in the discharge, the number of injected tungsten atoms is approximately $8.6 \times 10^{16}$. The discharge is maintained by three negative-ion-source-based NBIs (n-NBI\#1\#3) with energy of $180 \mathrm{keV}$ during 3.6-6.2 s and two positive-ion-source-based NBIs (p-NBI\#4-\#5) with energy of $40 \mathrm{keV}$ during $6.2-8.2 \mathrm{~s}$, as shown in Fig. 1 (a). Lineaveraged electron density, $n_{\mathrm{e}}$, central electron temperature, $T_{\mathrm{e} 0}$, total radiation power, $P_{\mathrm{rad}}$, and plasma stored energy, $W_{\mathrm{p}}$, are shown in Figs. 1 (b) - 1 (e), respectively. After tungsten pellet injection, $n_{\mathrm{e}}$ and $P_{\text {rad }}$ quickly increase, while $T_{\mathrm{e} 0}$ slowly decreases and $W_{\mathrm{p}}$ stays almost constant. The $T_{\mathrm{e}}$ and $n_{\mathrm{e}}$ profiles at different discharge timings during $t=4.3-5.2 \mathrm{~s}$ are shown in Figs. 2 (a) and 2 (b), respectively. Data at $t=4.3 \mathrm{~s}$ indicates $T_{\mathrm{e}}$ and $n_{\mathrm{e}}$ profiles just before the tungsten pellet injection. Although the $T_{\mathrm{e}}$ de-

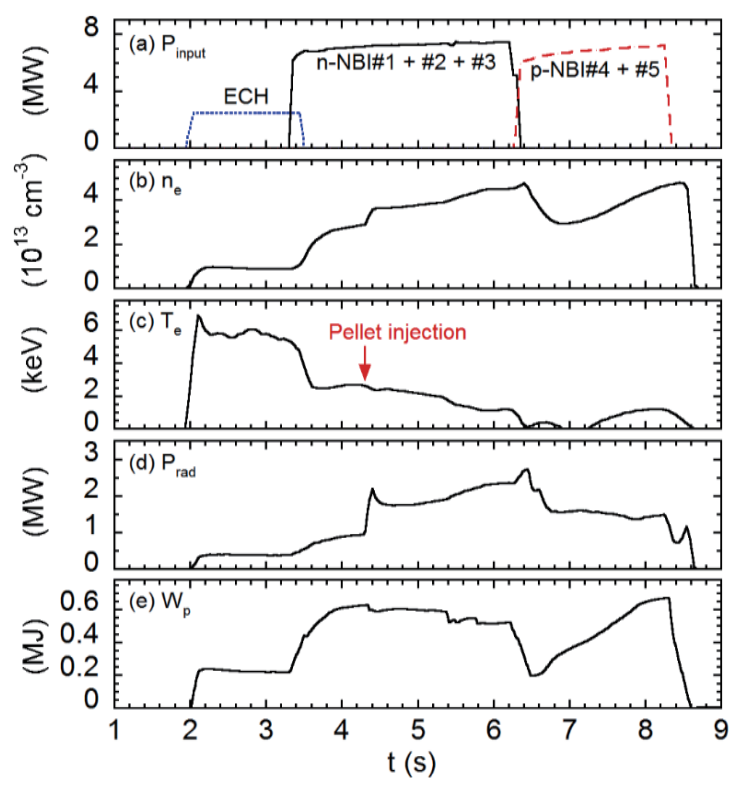

Fig. 1 Time behaviors of (a) port-through NBI power, (b) lineaveraged electron density, (c) central electron temperature, (d) total radiation power and (e) plasma stored energy. A coaxial pellet with tungsten wire size of $0.05 \mathrm{~mm}^{\phi} \times 0.7 \mathrm{~mm}^{\mathrm{L}}$ is injected at $t=4.33 \mathrm{~s}$.
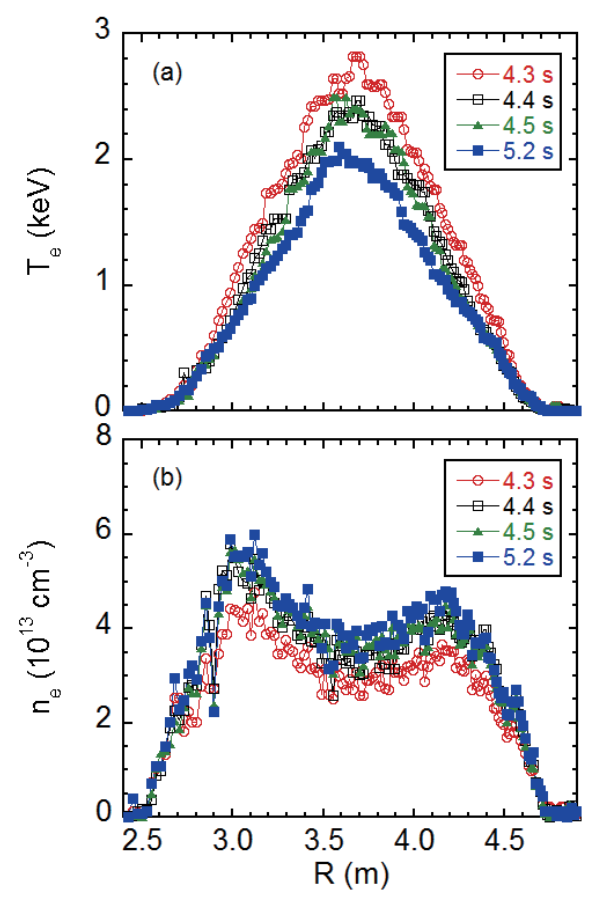

Fig. 2 Temporal evolutions of radial profiles of (a) electron temperature and (b) electron density just before $(t=4.3 \mathrm{~s})$ and after (4.4-5.2 s) the pellet injection.

creases and the $n_{\mathrm{e}}$ increases after the tungsten pellet injection, both radial profiles are almost unchanged. It indicates that the tungsten injection does not disturb so much the discharge condition.

The tungsten spectra at wavelength range of 10 - 

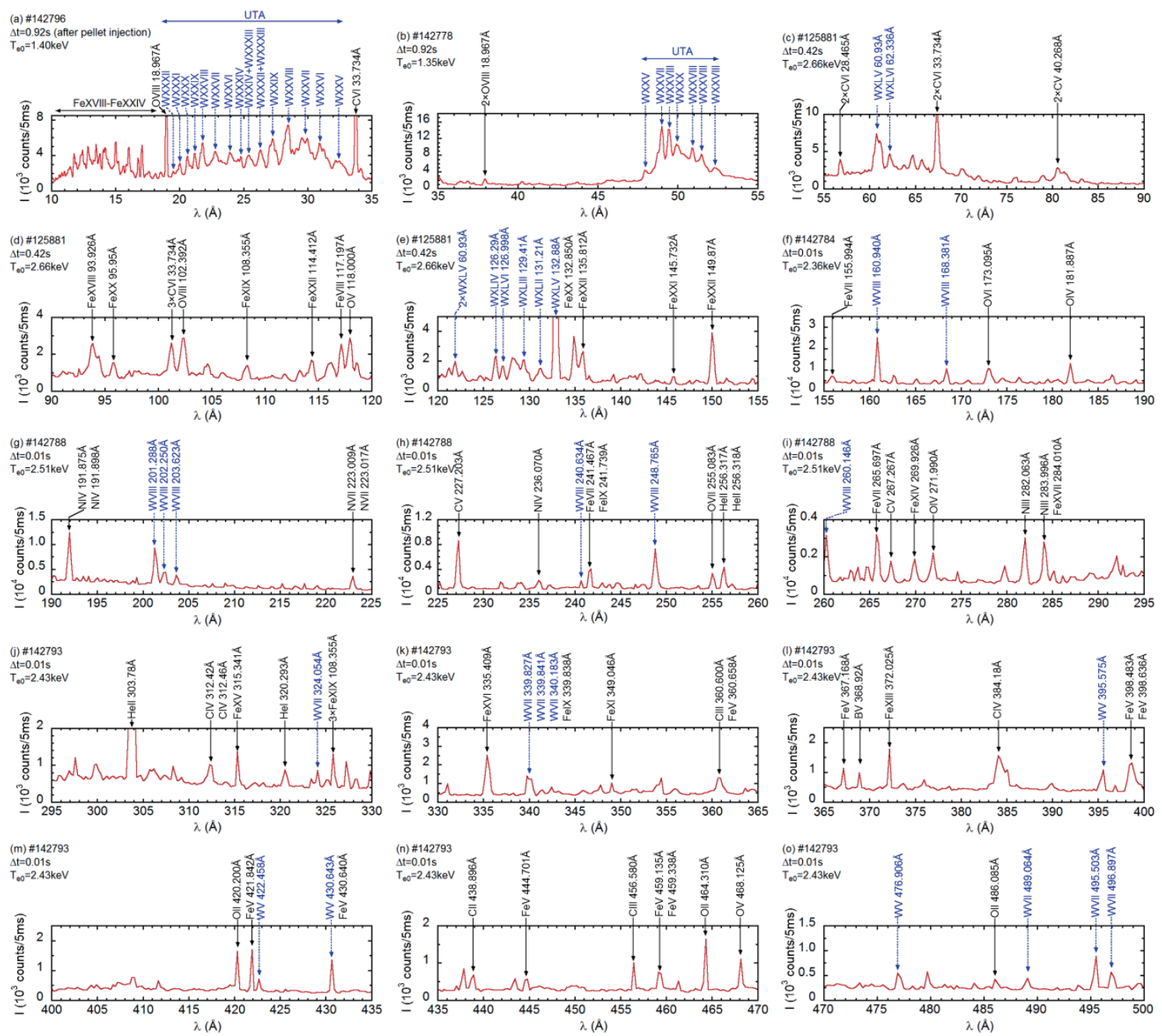

Fig. 3 EUV spectra of tungsten emission lines observed in LHD at (a) $10-35 \AA$, (b) 35 - $55 \AA$, (c) 55 - $90 \AA$, (d) 90 - $120 \AA$, (e) 120 - $155 \AA$, (f) $155-190 \AA$, (g) $190-225 \AA$, (h) $225-260 \AA$, (i) $260-295 \AA$, (j) $295-330 \AA$, (k) $330-365 \AA$, (l) $365-400 \AA$, (m) $400-435 \AA$, (n) $435-470 \AA$ and (o) $470-500 \AA$.

$500 \AA ̊$ observed with EUV_Short and EUV_Long spectrometers are carefully identified based on NIST atomic spectra database and previous studies $[8,15,16]$, as shown in Figs. 3 (a)-3(o). The shot number, central electron temperature and data acquisition timing after pellet injection are indicated for each spectrum. The spectra in Figs. 3(a) and 3(b) are measured by EUV_Short with spectral resolution of $0.051 \AA$ at $\lambda=25 \AA$ and $0.067 \AA$ at $\lambda=45 \AA$. The spectra in Figs. 3 (c) - 3 (o) are measured by EUV_Long with spectral resolution of $0.149 \AA$ at $\lambda=$ $105 \AA$ and $0.270 \AA$ at $\lambda=450 \AA$. Therefore, the uncertainty in the spectral wavelength determination is estimated to be $0.005 \AA$ for short wavelength range and $0.015-0.03 \AA$ for long wavelength range of which the values are calculated as one tenth of the spectral resolution. In Fig. 3, the tungsten line emissions are denoted with dotted arrows and line emissions from other impurity ions are denoted with solid arrows. The wavelength scale in abscissa of Fig. 3 is determined by linear interpolation and extrapolation based on the accurate wavelengths of well-known transitions in C,
$\mathrm{O}, \mathrm{N}$ and Fe impurity ions.

Two tungsten unresolved transition arrays (UTAs) appear at wavelength ranges of $19-35 \AA$ and $48-53 \AA$ as shown in Figs. 3 (a) and 3 (b), respectively. The UTA spectra are replotted in detail against different temperatures of $T_{\mathrm{e} 0}=0.42,0.87,1.63,1.94$ and $2.45 \mathrm{keV}$ as shown in Figs. 4 (a) - 4 (e), respectively. The UTA spectra composing of many tungsten ions largely move to shorter wavelength range with increase in $T_{\mathrm{e}}$ showing an increase in the ionization stage of tungsten ions and finally almost disappear when the $T_{\mathrm{e}}$ is sufficiently high (see Fig. $4(\mathrm{e})$ ). As one can see from the figures, a simple spectral identification of the UTA is quite difficult. In the present study, therefore, the UTA spectra are excluded from a summary table described later. Recently, on the other hand, the UTA spectrum at $19-35 \AA$ is attempted to understand by superposing two UTA CoBIT spectra [8]. A model calculation is also carried out to explain the LHD UTA spectrum [17]. In addition, the ionization stage of tungsten ions is determined at each wavelength interval of the UTA based on the 


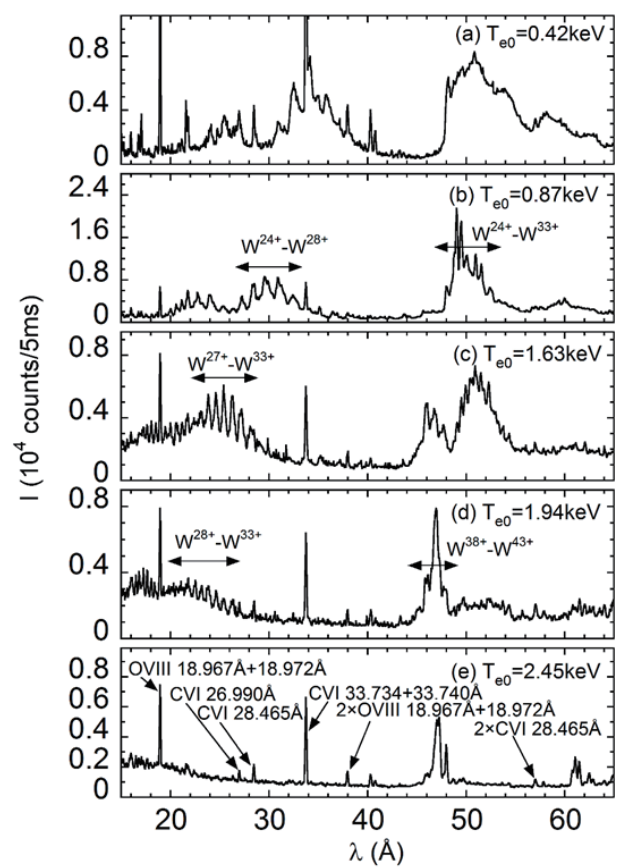

Fig. 4 Tungsten UTA spectra at central electron temperatures of (a) 0.42 , (b) 0.87 , (c) 1.63 , (d) 1.94 and (e) $2.45 \mathrm{keV}$.

profile measurement [16].

Tungsten line emissions from highly ionized ions are observed at $T_{\mathrm{e} 0}=2.66 \mathrm{keV}$, as shown in Figs. 3 (c) and 3 (e). These spectra are taken at $0.42 \mathrm{~s}$ after the pellet injection. The wavelength of a line emission identified as $\mathrm{W}^{44+}$ located at $60.93 \AA$ [18] is re-examined and determined to be $60.87 \AA$ in this study as shown in Fig. 3 (c), while the line emission is clearly broadened. It is investigated in a previous experimental work [19] that the $\mathrm{W}^{44+}$ line is blended with $\mathrm{W}^{42+}$ and $\mathrm{W}^{43+}$ line emissions. A line emission theoretically predicted as $\mathrm{W}^{45+}$ at $62.187 \AA$ [18] is identified at a wavelength of $62.188 \AA$ in this study, where the uncertainty in wavelength determination is $\sim 0.007 \AA$, while it is observed at $62.336 \AA$ in a previous experimental work [18]. This emission line is also blended with tungsten emissions in lower ionization stages [19]. The blended line is estimated to be $\mathrm{W}^{39+}$ at $62.366 \AA$ in EBIT result [18] and to be $\mathrm{W}^{44+}$ in HULLAC code [8]. Three line emissions measured at $126.29 \AA, 126.998 \AA$, and 131.21 $\AA$ are identified as a single ionization of $\mathrm{W}^{43+}, \mathrm{W}^{45+}$, and $\mathrm{W}^{41+}$, respectively $[19,20]$. A line emission measured at $129.45 \AA$ in Fig. 3 (e) is identified as $\mathrm{W}^{42+}[20,21]$. A line emission measured at $132.88 \AA$ is identified as $\mathrm{W}^{44+}$ [20,21]. It can be easily found because the intensity is very strong.

In the present identification mentioned above the reliability is also checked with the radial profile measured along the vertical direction. As an example of the profile measurement, vertical profiles of $\mathrm{W}^{43+}(126.05 \AA$ $\leq \lambda \leq 126.37 \AA), \mathrm{W}^{41+}(130.93 \AA \leq \lambda \leq 131.25 \AA)$ and $\mathrm{W}^{44+}(132.72 \AA \leq \lambda \leq 133.06 \AA)$ obtained with EUV_Long2 are plotted in Fig. 5. The emission lines at $126.05-126.37 \AA$ and $130.93-131.25 \AA$ are found not to

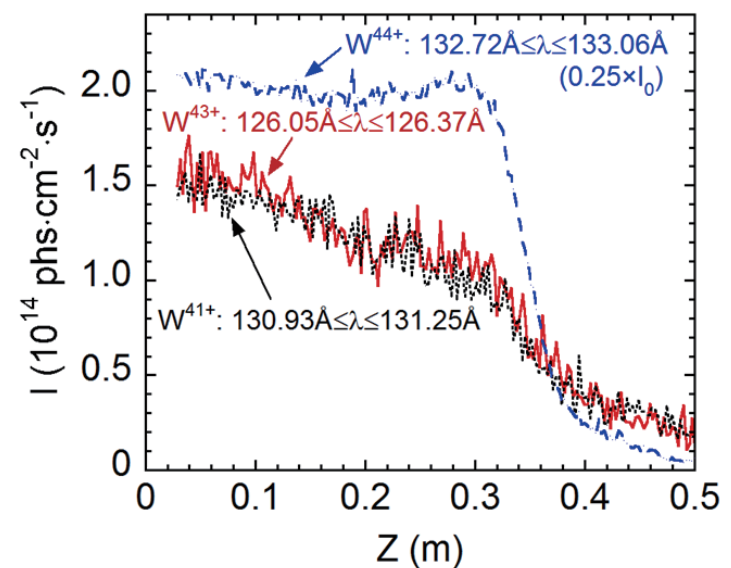

Fig. 5 Vertical profiles of $\mathrm{W}_{\text {XLII }}\left(\mathrm{W}^{41+}\right)$ (dotted line: $130.93 \AA$ $\leq \lambda \leq 131.25 \AA$ ), $\mathrm{W}_{\mathrm{XLIV}}\left(\mathrm{W}^{43+}\right)$ (solid line: $126.05 \AA \leq$ $\lambda \leq 126.37 \AA$ ) and $\mathrm{W}_{\mathrm{XLV}}\left(\mathrm{W}^{44+}\right)$ (dashed line: $132.72 \AA$ $\leq \lambda \leq 133.06 \AA)$. The $\mathrm{W}_{\mathrm{XLV}}$ is blended with $\mathrm{Fe}_{\mathrm{XX}}$ at $132.850 \AA$.

be blended with emission lines from intrinsic impurities in the LHD plasma, due to the centrally peaked profile. However, the vertical profile extracted at $132.72-133.06 \AA$ is clearly different having a shoulder at $Z=0.3 \mathrm{~m}$. The blended line is estimated to be $\mathrm{Fe}^{19+}$ at $132.850 \AA$ A because the $T_{\mathrm{e}}$ at the shoulder position is close to the ionization energy of $\mathrm{Fe}^{19+}$ ions.

A lot of EUV line emissions from low-ionized tungsten ions of $\mathrm{W}^{4+}, \mathrm{W}^{6+}$ and $\mathrm{W}^{7+}$, are clearly observed at long wavelength range of 155 - $500 \AA$ just after the tungsten pellet injection. A time duration at which such line emissions can be observed with relatively strong intensities is very short, e.g. $15 \mathrm{~ms}$ corresponding to $3 \mathrm{CCD}$ frames. Until now $\mathrm{W}_{\mathrm{VIII}}$ spectra are observed at $175-275 \AA$ in MTIM tokamak without identification due to a poor spectral resolution of $5 \AA$ at [22]. The $\mathrm{W}_{\mathrm{VIII}}$ spectra are also studied in EBIT device and calculation [23-25]. In LHD, $\mathrm{W}_{\mathrm{VIII}}$ lines are clearly found at 155-295 $\mathrm{A}$ in Figs. 3 (f) - 3 (i). However, those spectra are entirely different from EBIT spectra. The reason seems to originate in a different density between two devices. There are many data on lowionized tungsten in the NIST atomic spectra database [15]. In practice, most of these data originate in the spark experiment [26-29] and the tungsten spectra observed from the spark plasma with spectral resolution of $0.01 \AA$ are similar to the LHD spectra.

The $\mathrm{W}_{\mathrm{V}}$ and $\mathrm{W}_{\mathrm{VII}}$ lines can be identified at $295-500 \AA$ in Figs. $3(\mathrm{j})-3(\mathrm{o})$. The $\mathrm{W}_{\mathrm{VII}}$ spectra are mainly studied at 180 - $450 \AA$ in the SSPX spheromak and calculation due to less data from EBIT [25-27]. However, the spectra are also quite different from LHD spectra due to the same reason as EBIT. A study is also done at $180-450 \AA$ in spark device on $\mathrm{W}_{\mathrm{VII}}[26,27]$ and $\mathrm{W}_{\mathrm{V}}[28,29]$. The $\mathrm{W}_{\mathrm{V}}$ and $\mathrm{W}_{\mathrm{VII}}$ spectra from the spark are also identical to the LHD spectra.

The tungsten emission lines observed at $10-500 \AA$ in the present study are summarized in Table 1 except for the 
Table 1 Summary of spectral identification of tungsten line emissions. The first column shows tungsten ionization stage and the second and third columns give the wavelengths of line emissions from NIST database and the present observation, respectively. Discrepancy between $\lambda_{\text {NIST }}$ and $\lambda_{\text {obs }}$ and relative spectral intensity are shown in the fourth and fifth columns, respectively. The status in the sixth column of 'Single' or 'Blended' means the emission line consists of a single ionization stage or is blended with other emission lines.

\begin{tabular}{|c|c|c|c|c|c|}
\hline $\mathrm{W}^{\mathrm{q}^{+}}$ & $\lambda_{\mathrm{NIST}}(\AA)$ & $\lambda_{\text {obs }}(\AA)$ & $\begin{array}{c}\lambda_{\mathrm{NIST}}-\lambda_{\mathrm{obs}} \\
(\AA)\end{array}$ & $\begin{array}{c}\text { Intensity } \\
\text { (counts } / 5 \mathrm{~ms} \text { ) }\end{array}$ & Status \\
\hline \multirow{4}{*}{$\mathrm{WV}\left(\mathrm{W}^{4+}\right)$} & 395.575 & 395.571 & 0.004 & 1077 & Single \\
\hline & 422.458 & 422.421 & 0.037 & 3523 & Single \\
\hline & 430.643 & 430.629 & 0.014 & 1355 & $\begin{array}{c}\text { Blended } \\
\left(\mathrm{Fe}^{4+}\right)\end{array}$ \\
\hline & 476.906 & \begin{tabular}{|l|}
476.919 \\
\end{tabular} & -0.013 & 553 & Single \\
\hline \multirow{7}{*}{ WVII $\left(\mathrm{W}^{6+}\right)$} & 324.054 & 324.098 & -0.044 & 866 & Single \\
\hline & 339.827 & 339.749 & 0.078 & 1407 & Blended \\
\hline & 339.841 & 339.992 & -0.151 & 1233 & Blended \\
\hline & 340.183 & 340.235 & -0.052 & 1243 & Blended \\
\hline & 489.064 & 489.099 & -0.035 & 445 & Single \\
\hline & 495.503 & 495.509 & -0.006 & 889 & Single \\
\hline & 496.897 & 496.906 & -0.009 & 564 & Single \\
\hline \multirow{8}{*}{ WVIII $\left(\mathrm{W}^{7+}\right)$} & 160.940 & 160.998 & -0.058 & 26330 & Single \\
\hline & 168.381 & 168.405 & -0.024 & 10560 & Single \\
\hline & 201.288 & 201.266 & 0.022 & 9240 & Single \\
\hline & 202.250 & 202.249 & 0.001 & 4341 & Single \\
\hline & 203.623 & 203.630 & -0.007 & 3864 & Single \\
\hline & 240.634 & 240.667 & -0.033 & 2180 & Single \\
\hline & 248.765 & 248.765 & 0 & 7307 & Single \\
\hline & 260.146 & 260.241 & -0.095 & 3148 & Single \\
\hline WXLII $\left(\mathrm{W}^{41+}\right)$ & 131.21 & 131.25 & -0.04 & 1522 & Single \\
\hline WXLIII $\left(\mathrm{W}^{42+}\right)$ & 129.41 & 129.45 & -0.04 & 2084 & Blended \\
\hline WXLIV $\left(\mathrm{W}^{43+}\right)$ & 126.29 & 126.21 & 0.08 & 1651 & Single \\
\hline \multirow{2}{*}{ WXLV $\left(\mathrm{W}^{44+}\right)$} & 60.93 & 60.87 & 0.06 & 7387 & $\begin{array}{c}\text { Blended } \\
\left(\mathrm{W}^{42+}, \mathrm{W}^{43+}\right)\end{array}$ \\
\hline & 132.88 & 132.90 & -0.02 & 16051 & $\begin{array}{c}\text { Blended } \\
\left(\mathrm{Fe}^{19+}\right)\end{array}$ \\
\hline \multirow{2}{*}{ WXLVI $\left(\mathrm{W}^{45+}\right)$} & 126.998 & 127.017 & -0.019 & 1752 & Single \\
\hline & 62.336 & 62.188 & 0.148 & 4707 & Blended \\
\hline
\end{tabular}

UTA lines. In Table 1, the first column shows the tungsten ionization stage, and the second and third columns give the wavelengths from NIST database and the present observation, respectively. As described above the NIST data are based on identification results from the spark experiment. Discrepancies between $\lambda_{\mathrm{NIST}}$ and $\lambda_{\mathrm{obs}}$ indicated in the fourth column are obviously smaller than $0.1 \AA$ for all observed emission lines except for two lines at $\lambda_{\text {obs }}=$ $62.188 \AA$ and $\lambda_{\text {obs }}=339.992 \AA$. The reason is due to a large influence from blended lines. The intensity at the spectral peak is shown in the fifth column. The $\mathrm{W}_{\mathrm{VIII}}$ line at $\lambda_{\mathrm{obs}}$ $=160.998 \AA$ is found to be the strongest in the wavelength range of $155-500 \AA$. The $\mathrm{W}_{\mathrm{V}}$ and $\mathrm{W}_{\mathrm{VII}}$ intensities are relatively weak and many lines are blended with other lines. The status of observed tungsten lines is denoted in the sixth column. In the case of 'Blended' ionization stages of the blended lines are also indicated.

\section{Summary}

Tungsten spectra are observed at wavelength range of 10 - $500 \AA$ by injecting a coaxial tungsten impurity pellet in LHD. Two tungsten UTAs are observed at wavelength range of $10-55 \AA$. The UTA at $19-35 \AA$ moves toward shorter wavelength side with electron temperature and the UTA at $48-53 \AA$ consisting of $\mathrm{W}^{24+}-\mathrm{W}^{33+}$ ions gradually disappear with electron temperature. The UTA almost disappear at sufficiently high-temperature phase, e.g. $T_{\mathrm{e} 0}=$ $2.66 \mathrm{keV}$ and line emissions from highly ionized tungsten ions begin to be observed at 55 - $155 \AA$. Blended line emissions are examined by measuring the radial profile. A lot of line emissions from low-ionized tungsten ions of $\mathrm{W}^{4+}$, $\mathrm{W}^{6+}$ and $\mathrm{W}^{7+}$ are clearly observed at $155-500 \AA$ just after the tungsten pellet injection and identified based on the NIST database. All these line emissions are observed for the first time in the toroidal device, while the NIST data are based on the result from spark plasmas. The tungsten emission lines observed at $10-500 \AA$ are summarized in the table except for the UTA lines. The present identification shows a good agreement with the NIST data base. In particular, tungsten spectra from low-ionized ions observed for the first time in the toroidal device will contribute to the tungsten impurity diagnostics in fusion research in addition to the accuracy improvement of atomic structure modeling.

\section{Acknowledgments}

The authors thank all the members of the LHD experiment group for their cooperation including technical supports. This work was partly supported by LHD project (NIFS17ULPP010) and Grant-in-Aid for Scientific Research (B) No. 16H04088.

[1] R. Neu et al., Nucl. Fusion 45, 209 (2005).

[2] J. Roth et al., Plasma Phys. Control. Fusion 50, 103001 (2008).

[3] J.W. Coenen et al., J. Nucl. Mater. 463, 78 (2015).

[4] A. Geier, H. Maier and R. Neu, Plasma Phys. Control. Fusion 44, 2091 (2002).

[5] R. Dux et al., J. Nucl. Mater. 390-391, 858 (2009).

[6] G.J. Van Rooij et al., J. Nucl. Mater. 438, S42 (2013).

[7] T. Oishi, S. Morita, X. Huang, H. Zhang, M. Goto and the LHD Experimental Group, Phys. Scr. 91, 025602 (2016).

[8] S. Morita et al., AIP Conf. Proc. 1545, 143 (2013).

[9] T. Nakano and the JT-60 Team, J. Nucl. Mater. 415, S327 (2011).

[10] R. Barnsley, the Decennial IAEA Technical Meeting on Atomic, Molecular and Plasma-Material Interaction Data for Fusion Science and Technology, Daejeon, South Korea, December 2014. https://www-amdis.iaea.org/meetings/ AMPMI14/Presentations/AMPMI-2014-12-15-TalkBarnsley-ITER-2by4.pdf

[11] X. Huang, S. Morita, T. Oishi, M. Goto and H. Zhang, Rev. Sci. Instrum. 85, 11E818 (2014).

[12] M.B. Chowdhuri, S. Morita and M. Goto, Appl. Opt. 47, 135 (2008).

[13] M.B. Chowdhuri, S. Morita and M. Goto, Rev. Sci. Instrum. 78, 023501 (2007).

[14] C. Dong, S. Morita, M. Goto and H. Zhou, Rev. Sci. Instrum. 81, 033107 (2010).

[15] A. Kramida et al., NIST Atomic Spectra Database 
(ver. 5.5.1) [Online] Available: http://physics.nist.gov/asd [2 November 2017] (2017).

[16] Y. Liu, S. Morita, X. Huang, T. Oishi, M. Goto and H. Zhong, J. Appl. Phys. 122, 233301 (2017).

[17] I. Murakami, H.A. Sakaue, C. Suzuki, D. Kato, M. Goto, N. Tamura, S. Sudo, S. Morita and LHD Experiment Group, Nucl. Fusion 55, 093016 (2015).

[18] S.B. Utter, P. Beiersdorfer and E. Träbert, Can. J. Phys. 80, 1503 (2002).

[19] T. Pütterich, R. Neu, R. Dux, A.D. Whiteford, M.G. O'Mullane and the ASDEX Upgrade Team, Plasma Phys. Control. Fusion 50, 085016 (2008).

[20] T. Pütterich, R. Neu, C. Biedermann, R. Radtke and ASDEX Upgrade Team, J. Phys. B: At. Mol. Opt. Phys. 38, 3071 (2005).

[21] Y. Ralchenko, J. Reader, J.M. Pomeroy, J.N. Tan and J.D.
Gillaspy, J. Phys. B: At. Mol. Opt. Phys. 40, 3861 (2007).

[22] G. Veres, J.S. Bakos and B. Kardon, J. Quant. Spectrosc. Radiat. Transf. 56, 295 (1996).

[23] J. Deprince and P. Quinet, Atoms 3, 299 (2015).

[24] J. Clementson, T. Lennartsson and P. Beiersdorfer, Atoms 3, 407 (2015).

[25] J. Clementson, P. Beiersdorfer, E.W. Magee, H.S. Mclean and R.D. Wood, J. Phys. B: At. Mol. Opt. Phys. 43, 144009 (2010).

[26] J. Sugar and V. Kaufman, Phys. Rev. A 12, 994 (1975).

[27] J.F. Wyart, V. Kaufman and J. Sugar, Phys. Scr. 23, 1069 (1981).

[28] S.S. Churilov, R.R. Kildiyarova and Y.N. Joshi, Can. J. Phys. 74, 145 (1996).

[29] R.R. Kildiyarova, S.S. Churilov, Y.N. Joshi and A.N. Ryabtsev, Phys. Scr. 53, 454 (1996). 